PROCEEDINGS OF THE

AMERICAN MATHEMATICAL SOCIETY

Volume 135, Number 8, August 2007, Pages 2443-2451

S 0002-9939(07)08582-6

Article electronically published on April 10, 2007

\title{
WEYL'S THEOREM FOR PERTURBATIONS OF PARANORMAL OPERATORS
}

\author{
PIETRO AIENA AND JESÚS R. GUILLEN
}

(Communicated by Joseph A. Ball)

\begin{abstract}
A bounded linear operator $T \in L(X)$ on a Banach space $X$ is said to satisfy "Weyl's theorem" if the complement in the spectrum of the Weyl spectrum is the set of all isolated points of the spectrum which are eigenvalues of finite multiplicity. In this paper we show that if $T$ is a paranormal operator on a Hilbert space, then $T+K$ satisfies Weyl's theorem for every algebraic operator $K$ which commutes with $T$.
\end{abstract}

\section{Preliminaries AND NOTAtion}

Let $L(X)$ be the Banach algebra of all bounded linear operators on an infinitedimensional complex Banach space $X$. If $T \in L(X)$ set $\alpha(T):=\operatorname{dim} \operatorname{ker} T$, the dimension of the kernel, and $\beta(T):=\operatorname{codim} T(X)$, the codimension of the range. The class of all upper semi-Fredholm operators is defined as the set $\Phi_{+}(X)$ of all $T \in L(X)$ such that $\alpha(T)<\infty$ and $T(X)$ is closed. The class of all lower semi-Fredholm operators is defined as the set $\Phi_{-}(X)$ of all $T \in L(X)$ such that $\beta(T)<\infty$. The class of all semi-Fredholm operators is denoted by $\Phi_{ \pm}(X)$, while by $\Phi(X):=\Phi_{+}(X) \cap \Phi_{-}(X)$ we shall denote the class of all Fredholm operators. The index of $T \in \Phi_{ \pm}(X)$ is defined by ind $(T):=\alpha(T)-\beta(T)$. The other two classical quantities associated with a linear operator $T$ are the ascent $p:=p(T)$, defined as the smallest nonnegative integer $p$ (if it does exist) such that $\operatorname{ker} T^{p}=\operatorname{ker} T^{p+1}$, and the descent $q:=q(T)$, defined as the smallest nonnegative integer $q$ (if it does exists) such that $T^{q}(X)=T^{q+1}(X)$. It is well known that if $p(\lambda I-T)$ and $q(\lambda I-T)$ are both finite, then $p(\lambda I-T)=q(\lambda I-T)$ and $\lambda$ is a pole of the function resolvent $\lambda \rightarrow(\lambda I-T)^{-1}$, in particular an isolated point of the spectrum $\sigma(T)$; see Proposition 38.3 and Proposition 50.2 of Heuser 14. The class of Weyl operators is defined by

$$
W(X):=\{T \in \Phi(X): \operatorname{ind} T=0\},
$$

while the class of all Browder operators is defined by

$$
B(X):=\{T \in \Phi(X): p(T), q(T)<\infty\} .
$$

Obviously $B(X) \subseteq W(X)$. The Weyl spectrum and the Browder spectrum of $T \in$ $L(X)$ are defined by

$$
\sigma_{\mathrm{w}}(T):=\{\lambda \in \mathbb{C}: \lambda I-T \notin W(X)\}
$$

Received by the editors June 7, 2005 and, in revised form, November 21, 2005.

2000 Mathematics Subject Classification. Primary 47A10, 47A11; Secondary 47A53, 47A55.

Key words and phrases. Weyl's theorem, localized SVEP, paranormal operators. 
and

$$
\sigma_{\mathrm{b}}(T):=\{\lambda \in \mathbb{C}: \lambda I-T \notin B(X)\} .
$$

Recall that $T \in L(X)$ is said to be a Riesz operator if $\lambda I-T \in \Phi(X)$ for all $\lambda \in \mathbb{C} \backslash\{0\}$. Evidently, every quasi-nilpotent operator is a Riesz operator. It is well known that if $Q$ is a quasi-nilpotent operator commuting with $T$, then

$$
T \text { invertible } \Leftrightarrow T+Q \text { invertible, }
$$

so that $\sigma(T+Q)=\sigma(T)$. Moreover, if $R$ is a Riesz operator commuting with $T$, then $T \in W(X)$ if and only if $T+R \in W(X)$ (see Lemma 2.2 of [17]), and this obviously implies the equality $\sigma_{\mathrm{w}}(T+Q)=\sigma_{\mathrm{w}}(T)$ for every quasi-nilpotent operator $Q$ commuting with $T$. It is well known that a similar result holds for $\sigma_{\mathrm{b}}(T)$, i.e., $\sigma_{\mathrm{b}}(T+Q)=\sigma_{\mathrm{b}}(T)$, for every quasi-nilpotent operator $Q$ commuting with $T$ ([17, Proposition 4]).

An operator $T \in L(X)$ is said to admit a generalized Kato decomposition, abbreviated GKD, if there exists a pair of $T$-invariant closed subspaces $(M, N)$ such that $X=M \oplus N$, the restriction $T_{M}:=T \mid M$ is semi-regular (i.e., $T(M)$ is closed and $\operatorname{ker} T_{M} \subseteq\left(T_{M}\right)^{n}(M)$ for all $n \in \mathbb{N}$ ), while $T_{N}:=T \mid N$ is quasi-nilpotent. A relevant case is obtained if we assume in the definition above that $T_{N}$ is nilpotent. In this case $T$ is said to be of Kato type; see for details Chapter 1 of [1. A classical result of Kato establishes that every semi-Fredholm operator is of Kato type (see [1. Theorem 1.62]).

The single-valued extension property was introduced in the early years of local spectral theory by Dunford ([10, [11) and plays an important role in spectral theory; see the recent monographs by Laursen and Neumann [15], or by Aiena [1]. We shall consider the following local version of this property introduced by Finch 12 .

Definition 1.1. Let $X$ be a complex Banach space and $T \in L(X)$. The operator $T$ is said to have the single-valued extension property at $\lambda_{0} \in \mathbb{C}$ (abbreviated SVEP at $\lambda_{0}$ ), if for every open $\operatorname{disc} U$ of $\lambda_{0}$, the only analytic function $f: U \rightarrow X$ which satisfies the equation $(\lambda I-T) f(\lambda)=0$ for all $\lambda \in U$ is the function $f \equiv 0$.

An operator $T \in L(X)$ is said to have SVEP if $T$ has SVEP at every point $\lambda \in \mathbb{C}$.

Evidently, an operator $T \in L(X)$ has SVEP at every point of the resolvent $\rho(T):=\mathbf{C} \backslash \sigma(T)$. Moreover, from the identity theorem for analytic functions it easily follows that $T \in L(X)$ has SVEP at every point of the boundary $\partial \sigma(T)$ of the spectrum $\sigma(T)$. This implies, in particular, that both $T$ and $T^{*}$ have SVEP at every isolated point of $\sigma(T)=\sigma\left(T^{*}\right)$. The SVEP is inherited by the restrictions to closed invariant subspaces, i.e., if $T \in L(X)$ has the SVEP at $\lambda_{0}$ and $M$ is a closed $T$-invariant subspace, then $T \mid M$ has SVEP at $\lambda_{0}$. Furthermore, if $T$ has SVEP, then $\mu I-T$ has SVEP for every $\mu \in \mathbb{C}$.

The proof of the following result may be found in [1, Corollary 2.12].

Theorem 1.2. Suppose that $T$ has $S V E P$. If $Q$ is a quasi-nilpotent operator which commutes with $T$, then also $T+Q$ has $S V E P$.

The quasi-nilpotent part of $T$ is defined by

$$
H_{0}(T):=\left\{x \in X: \lim _{n \rightarrow \infty}\left\|T^{n} x\right\|^{\frac{1}{n}}=0\right\} .
$$


It is easily seen that ker $T^{m} \subseteq H_{0}(T)$ for every $m \in \mathbb{N}$. Moreover, $T$ is quasinilpotent if and only if $H_{0}(T)=X$ (see [1, Theorem 1.68]), while if $T$ is invertible, then $H_{0}(T)=\{0\}$.

If $T \in L(X)$, the analytic core $K(T)$ is the set of all $x \in X$ such that there exists a constant $c>0$ and a sequence of elements $x_{n} \in X$ such that $x_{0}=x, T x_{n}=x_{n-1}$, and $\left\|x_{n}\right\| \leq c^{n}\|x\|$ for all $n \in \mathbb{N}$. Note that both $H_{0}(T)$ and $K(T)$ generally are not closed linear subspaces (for details we refer to [1]) and $T(K(T))=K(T)$. We have

$$
H_{0}(\lambda I-T) \text { closed } \Rightarrow T \text { has SVEP at } \lambda ;
$$

see 3 .

A bounded operator $T \in L(X)$ on a Banach space $X$ is said to be paranormal if

$$
\|T x\|^{2} \leq\left\|T^{2} x\right\|\|x\| \text { holds for all } x \in X .
$$

There is a growing interest concerning paranormal operators [4, [14, §54], 6], [8] and subclasses of paranormal operators [13, 17], 2]. In fact, the class of paranormal operators properly contains a relevant number of Hilbert space operators, among them $p$-hyponormal operators, log-hyponormal operators, quasi-hyponormal operators $\left(\left(A^{*} A\right)^{2} \leq A^{* 2} A^{2}\right)$, and the class of operators satisfying the condition $|T|^{2} \leq\left|T^{2}\right|$; see [13] and [9]. Every paranormal operator on a Hilbert space has SVEP. This may be easily seen as follows: if $\lambda \neq 0$ and $\lambda \neq \mu$, then, by Theorem 2.6 of [6], we have $\|x+y\| \geq\|y\|$ whenever $x \in \operatorname{ker}(\mu I-T)$ and $y \in \operatorname{ker}(\lambda I-T)$. It then follows that if $U$ is an open disc and $f: U \rightarrow X$ is an analytic function such that $0 \neq f(z) \in \operatorname{ker}(z I-T)$ for all $z \in U$, then $f$ fails to be continuous at every $0 \neq \lambda \in U$.

Note that every paranormal operator $T$ is normaloid (i.e., $\|T\|=r(T), r(T)$ the spectral radius of $T$; see [14, Proposition 54.6]), so, if $T$ is a quasi-nilpotent paranormal operator, then $T=0$.

An operator $T \in L(X)$ for which there exists a complex nonconstant polynomial $h$ such that $h(T)$ is paranormal is said to be algebraically paranormal. Note that algebraic paranormality is preserved under translation by scalars and under restriction to closed invariant subspaces. Every algebraically paranormal operator has SVEP, since $h(T)$ has SVEP ([1, Theorem 2.40]).

\section{WEYL'S THEOREMS}

Let us write iso $K$ for the set of all isolated points of $K \subseteq \mathbb{C}$. For a bounded operator $T \in L(X)$ define

$$
\pi_{00}(T):=\{\lambda \in \text { iso } \sigma(T): 0<\alpha(\lambda I-T)<\infty\}
$$

and

$$
p_{00}(T):=\sigma(T) \backslash \sigma_{\mathrm{b}}(T)=\{\lambda \in \sigma(T): \lambda I-T \in \mathcal{B}(X)\},
$$

to be the set of all Riesz points in $\sigma(T)$. Clearly, $p_{00}(T) \subseteq \pi_{00}(T)$ for every $T \in L(X)$ and $p_{00}(T)=p_{00}(T+Q)$ for every quasi-nilpotent operator $Q$ commuting with $T$.

Following Coburn [7, we say that Weyl's theorem holds for $T \in L(X)$ if

$$
\sigma(T) \backslash \sigma_{\mathrm{w}}(T)=\pi_{00}(T) .
$$


Theorem 2.1 ([2]). If $T \in L(X)$, then the following assertions are equivalent:

(i) Weyl's theorem holds for $T$.

(ii) $T$ has SVEP at every point $\lambda \notin \sigma_{\mathrm{w}}(T)$ and $\pi_{00}(T)=p_{00}(T)$.

Let $\mathcal{P}_{0}(X), X$ a Banach space, denote the class of all operators $T \in L(X)$ such that there exists $p:=p(\lambda) \in \mathbb{N}$ for which

$$
H_{0}(\lambda I-T)=\operatorname{ker}(\lambda I-T)^{p} \quad \text { for all } \lambda \in \pi_{00}(T) .
$$

Theorem 2.2. $T \in \mathcal{P}_{0}(X)$ if and only if $p_{00}(T)=\pi_{00}(T)$. In particular, if $T$ has $S V E P$, then Weyl's theorem holds for $T$ if and only if $T \in \mathcal{P}_{0}(X)$.

Proof. Suppose $T \in \mathcal{P}_{0}(X)$ and $\lambda \in \pi_{00}(T)$. Then there exists $p \in \mathbb{N}$ such that $H_{0}(\lambda I-T)=\operatorname{ker}(\lambda I-T)^{p}$. Since $\lambda$ is isolated in $\sigma(T)$ then, by [1, Theorem 3.74],

$$
X=H_{0}(\lambda I-T) \oplus K(\lambda I-T)=\operatorname{ker}(\lambda I-T)^{p} \oplus K(\lambda I-T),
$$

from which we obtain

$$
(\lambda I-T)^{p}(X)=(\lambda I-T)^{p}(K(\lambda I-T))=K(\lambda I-T),
$$

so $X=\operatorname{ker}(\lambda I-T)^{p} \oplus(\lambda I-T)^{p}(X)$, which implies, by [1, Theorem 3.6], that $p(\lambda I-T)=q(\lambda I-T) \leq p$. By definition of $\pi_{00}(T)$ we know that $\alpha(\lambda I-T)<\infty$ and this implies by Theorem 3.4 of [1] that $\beta(\lambda I-T)$ is also finite. Therefore $\lambda \in p_{00}(T)$ and hence $\pi_{00}(T) \subseteq p_{00}(T)$. Since the opposite inclusion holds for every operator we then conclude that $p_{00}(T)=\pi_{00}(T)$.

Conversely, if $p_{00}(T)=\pi_{00}(T)$ and $\lambda \in \pi_{00}(T)$, then $p:=p(\lambda I-T)=q(\lambda I-T)<$ $\infty$. By Theorem 3.16 of [1] it then follows that $H_{0}(\lambda I-T)=\operatorname{ker}(\lambda I-T)^{p}$.

The last assertion is clear from Theorem 2.1.

In [18] Oudghiri studied the class $\mathcal{P}(X)$ of operators on Banach spaces for which there exists $p:=p(\lambda) \in \mathbb{N}$ such that

$$
H_{0}(\lambda I-T)=\operatorname{ker}(\lambda I-T)^{p} \quad \text { for all } \lambda \in \mathbb{C} .
$$

Evidently, if $T \in \mathcal{P}(X)$, then $T \in \mathcal{P}_{0}(H)$. By (2) we also see that condition (4) entails that $T$ has SVEP. Consequently, by Theorem 2.2, if $T \in \mathcal{P}(X)$, then Weyl's theorem holds for $T([18])$. Note that $\mathcal{P}(X)$ is a rather large class of operators, for instance it contains every generalized scalar operator [18, and this implies that Weyl's theorem holds for p-hyponormal, log-hyponormal, and M-hyponormal operators. The next example shows that paranormal operators on Hilbert spaces may fail property (4).

Example 2.3. Let $T$ be a quasi-hyponormal operator on a Hilbert space $H$. Then $p(\lambda I-T) \leq 1$ for all $\lambda \in \mathbb{C}$, every eigenvalue is an isolated point of $\sigma(T), 0$ is an eigenvalue and the corresponding eigenspace ker $T$ is not reducing; see $[5$ for details. Clearly, $p(T)=1$. Suppose that $T$ satisfies property (4). Then $H_{0}(T)=\operatorname{ker} T$, and since $0 \in$ iso $\sigma(T)$ by Theorem 3.74 of [1] we have $H=\operatorname{ker} T \oplus K(T)$, from which we obtain $T(H)=T(K(T))=K(T)$. Hence $H=\operatorname{ker} T \oplus T(H)$. Since $T(H)=K(T)$ is closed (it coincides with the range of the spectral projection associated with $\{0\}$, see [1. Theorem 3.74]), it then follows that $T(H)=\left(\operatorname{ker} T^{*}\right)^{\perp}$, so ker $T$ is reducing, a contradiction.

However, we have:

Theorem 2.4. If $T \in L(H)$ is algebraically paranormal, then every isolated point of the spectrum is a pole of the resolvent. Furthermore, $T \in \mathcal{P}_{0}(H)$. 
Proof. Note first that every quasi-nilpotent algebraically paranormal operator $T$ is nilpotent. In fact, suppose that $h$ is a polynomial for which $h(T)$ is paranormal. From the spectral mapping theorem we have $\sigma(h(T))=h(\sigma(T))=\{h(0)\}$, so $h(0) I-h(T)$ is quasi-nilpotent. Since $h(0) I-h(T)$ is paranormal, then $h(0) I-$ $h(T)=0$, and hence there are some $n \in \mathbb{N}$ and $\mu \in \mathbb{C}$, such that

$$
0=h(0) I-h(T)=\mu T^{m} \prod_{i=1}^{n}\left(\lambda_{i} I-T\right) \quad \text { with } \lambda_{i} \neq 0 .
$$

Since all $\lambda_{i} I-T$ are invertible it then follows that $T^{m}=0$.

We show now that for every isolated point $\lambda$ of $\sigma(T)$ we have $p(\lambda I-T)=$ $q(\lambda I-T)<\infty$, i.e., $\lambda$ is a pole of the resolvent. If $\lambda \in$ iso $\sigma(T)$, let $P$ denote the spectral projection associated with $\{\lambda\}, M:=K(\lambda I-T)=\operatorname{ker} P$ and $N:=$ $H_{0}(\lambda I-T)=P(X)$. Then, by the classical spectral decomposition, $(M, N)$ is a GKD for $\lambda I-T$. Since $\lambda I-T \mid N$ is quasi-nilpotent and algebraically paranormal, then $\lambda I-T \mid N$ is nilpotent and hence $\lambda I-T$ is of Kato type. The SVEP for $T$ and $T^{*}$ at $\lambda$ then implies by Theorem 3.16 and Theorem 3.17 of [1] that both $p(\lambda I-T)$ and $q(\lambda I-T)$ are finite; hence $\lambda$ is a pole of the resolvent. To show the equality $p_{00}(T)=\pi_{00}(T)$ it suffices to prove the inclusion $\pi_{00}(T) \subseteq p_{00}(T)$. If $\lambda \in \pi_{00}(T)$, then $0<\alpha(\lambda I-T)<\infty$, and the equality $p(\lambda I-T)=q(\lambda I-T)<\infty$ entails by [1. Theorem 3.4] that $\beta(\lambda I-T)=\alpha(\lambda I-T)<\infty$, so $\lambda \in \sigma(T) \backslash \sigma_{\mathrm{b}}(T)=p_{00}(T)$.

Weyl's theorem for algebraically paranormal operators $T$ on Hilbert spaces has been proved by Curto and Han 8 (this is also immediate from Theorem 2.2 and Theorem 2.4, once observed that $T$ has SVEP).

A bounded operator $T$ is said to be algebraic if there exists a nontrivial polynomial $q$ such that $q(T)=0$. From the spectral mapping theorem it easily follows that the spectrum of an algebraic operator is a finite set.

It is known that Weyl's theorem is not generally transmitted to perturbation of operators satisfying Weyl's theorem. In [19] Oudghiri proved that if $T$ has property (44) and $K$ is an algebraic operator commuting with $T$, then $T+K$ satisfies Weyl's theorem. Our main result extends Oudghiri's result to paranormal operators:

Theorem 2.5. Suppose that $T \in L(H)$ is paranormal, $K$ algebraic and $T K=K T$. Then Weyl's theorem holds for $T+K$.

The proof of Theorem 2.5 proceeds through some steps, stated below as lemmas. We first begin with a useful remark.

Remark 2.6. Suppose that for a linear operator $T$ we have $\alpha(T)<\infty$. Then $\alpha\left(T^{n}\right)<\infty$ for all $n \in \mathbb{N}$. This may be easily seen by an inductive argument. Suppose that $\operatorname{dim} \operatorname{ker} T^{n}<\infty$. Since $T\left(\operatorname{ker} T^{n+1}\right) \subseteq \operatorname{ker} T^{n}$, then the restriction $T_{0}:=T \mid \operatorname{ker} T^{n+1}: \operatorname{ker} T^{n+1} \rightarrow \operatorname{ker} T^{n}$ has kernel equal to $\operatorname{ker} T$ so the canonical mapping $\hat{T}: \operatorname{ker} T^{n+1} / \operatorname{ker} T \rightarrow \operatorname{ker} T^{n}$ is injective. Therefore we have $\operatorname{dim} \operatorname{ker} T^{n+1} / \operatorname{ker} T \leq \operatorname{dim} \operatorname{ker} T^{n}<\infty$, and since $\operatorname{dim} \operatorname{ker} T<\infty$ we then conclude that $\operatorname{dim} \operatorname{ker} T^{n+1}<\infty$.

Lemma 2.7. Suppose that $T \in L(X)$ and $N$ is nilpotent such that $T N=N T$. Then $T \in \mathcal{P}_{0}(X)$ if and only if $T+N \in \mathcal{P}_{0}(X)$.

Proof. Suppose that $N^{p}=0$. Observe first that without any assumption on $T$ we have

$$
\operatorname{ker} T \subseteq \operatorname{ker}(T+N)^{p} \quad \text { and } \operatorname{ker}(T+N) \subseteq \operatorname{ker} T^{p}
$$


The first inclusion in (5) is clear, since for $x \in \operatorname{ker} T$ we have

$$
(T+N)^{p} x=N^{p} x=0 .
$$

To show the second inclusion in (5) observe that if $x \in \operatorname{ker}(T+N)$, then $T^{p} x=$ $(-1)^{p} N^{p} x=0$.

Suppose now that $T \in \mathcal{P}_{0}(X)$, or equivalently $p_{00}(T)=\pi_{00}(T)$. We show first that $\pi_{00}(T)=\pi_{00}(T+N)$. Let $\lambda \in \pi_{00}(T)$. There is no harm if we suppose $\lambda=0$. From $\sigma(T+N)=\sigma(T)$ we see that $0 \in$ iso $(T+N)$. Since $0 \in \pi_{00}(T)$, then $\alpha(T)>0$ and hence by the first inclusion in (5) we have $\alpha(T+N)^{p}>0$ and this obviously implies that $\alpha(T+N)>0$. To show that $\alpha(T+N)<\infty$, observe that

$$
\operatorname{ker}(T+N) \subseteq \operatorname{ker} T^{p} \subseteq H_{0}(T) .
$$

The equality $p_{00}(T)=\pi_{00}(T)$ is equivalent to saying that $H_{0}(\lambda I-T)$ is finitedimensional for all $\lambda \in \pi_{00}(T)$ (see [1, Theorem 3.84]), and hence $H_{0}(T)$ is finitedimensional. Therefore $\alpha(T+N)<\infty$, so $0 \in \pi_{00}(T+N)$ and the inclusion $\pi_{00}(T) \subseteq \pi_{00}(T+N)$ is proved.

To show the opposite inclusion, assume that $0 \in \pi_{00}(T+N)$. Clearly, $0 \in$ iso $\sigma(T)=$ iso $\sigma(T+N)$. By assumption $\alpha(T+N)>0$, so the second inclusion in (5) entails that $\alpha\left(T^{p}\right)>0$ and this trivially implies that $\alpha(T)>0$. We also have $\alpha(T+N)<\infty$ and hence, by Remark 2.6. $\alpha(T+N)^{p}<\infty$. From the first inclusion in (5D) we then conclude that $\alpha(T)<\infty$. This shows that $0 \in \pi_{00}(T)$, so the equality $\pi_{00}(T)=\pi_{00}(T+N)$ is proved.

Finally, if $T \in \mathcal{P}_{0}(X)$, then $p_{00}(T+N)=p_{00}(T)=\pi_{00}(T)=\pi_{00}(T+N)$, so $T+N \in \mathcal{P}_{0}(X)$. Conversely, if $T+N \in \mathcal{P}_{0}(X)$ by symmetry we have

$$
p_{00}(T)=p_{00}(T+N)=\pi_{00}(T+N)=\pi_{00}((T+N)-N)=\pi_{00}(T),
$$

so the proof is complete.

From Lemma 2.7 and from the equalities (10) we easily obtain the following Oberai's result [16]: if $N$ is a nilpotent operator commuting with $T$, then

Define

$$
\text { Weyl's theorem holds for } T \Leftrightarrow \text { Weyl's theorem holds for } T+N \text {. }
$$

\section{Define}

$$
\pi_{\text {of }}(T):=\{\lambda \in \text { iso } \sigma(T): \alpha(\lambda I-T)<\infty\} .
$$

Lemma 2.8. Let $T \in L(X), X$ a Banach space. If $R$ is a Riesz operator that commutes with $T$, then

$$
\pi_{0 f}(T+R) \cap \sigma(T) \subseteq \text { iso } \sigma(T)
$$

If $T \in L(H)$ is paranormal and $N$ is a nilpotent operator commuting with $T$, then

$$
\pi_{0 \mathrm{f}}(T+N) \cap \sigma(T) \subseteq p_{00}(T) .
$$

Proof. The inclusion (7) has been proved in [19, Lemma 2.3].

Assume that $T$ is paranormal, $N$ is nilpotent commuting with $T$ and let $p \in \mathbb{N}$ be such that $N^{p}=0$. Let $\lambda \in \pi_{0 \mathrm{f}}(T+N) \cap \sigma(T)$. By Remark 2.6 we have $\alpha\left((\lambda I-(T+N))^{p}\right)<\infty$. From the first inclusion of (5) it then follows that $\alpha(\lambda I-T) \leq \alpha\left((\lambda I-(T+N))^{p}\right)<\infty$. Now, $\lambda \in$ iso $\sigma(T+N)=$ iso $\sigma(T)$ and $T$ is paranormal, so by Theorem $2.4 \lambda$ is a pole of the resolvent, or equivalently $0<p(\lambda I-T)=q(\lambda I-T)<\infty$. By Theorem 3.4 of [1] we then conclude that $\alpha(\lambda I-T)=\beta(\lambda I-T)<\infty$ and hence $\lambda \in p_{00}(T)$, as required.

Now we can give the proof of our main result. 
Proof of Theorem 2.5. Let $\sigma(K)=\left\{\mu_{1}, \ldots, \mu_{n}\right\}$. Denote by $P_{i}$ the spectral projection associated with $K$ and the spectral set $\left\{\mu_{i}\right\}$. Let $Y_{i}:=P_{i}(H)$ and $Z_{i}:=\operatorname{ker} P_{i}$. Then $H=Y_{i} \oplus Z_{i}$, the closed subspaces $Y_{i}$ and $Z_{i}$ are invariant under $T$ and $K$, and $\sigma\left(K \mid Y_{i}\right)=\left\{\mu_{i}\right\}$. Define $K_{i}:=K \mid Y_{i}$ and $T_{i}:=T \mid Y_{i}$. Clearly, the restrictions $T_{i}$ and $K_{i}$ commute for every $i=1,2, \ldots, n$ and

$$
\sigma(T+K)=\sigma\left(T_{i}+K_{i}\right) \cup \sigma\left((T+K) \mid Z_{i}\right) .
$$

We show first that $T_{i}+K_{i} \in \mathcal{P}_{0}\left(Y_{i}\right)$ for every $i=1,2, \ldots, n$. Let $h$ be a polynomial such that $h(K)=0$. Then $h\left(K_{i}\right)=h(K) \mid Y_{i}=0$, and from

$$
\{0\}=\sigma\left(h\left(K_{i}\right)\right)=h\left(\sigma\left(K_{i}\right)\right)=h\left(\left\{\mu_{i}\right\}\right)
$$

we obtain that $h\left(\mu_{i}\right)=0$. Write

$$
h(\mu)=\left(\mu_{i}-\mu\right)^{\nu} q(\mu) \quad \text { with } q\left(\mu_{i}\right) \neq 0 .
$$

Then $0=h\left(K_{i}\right)=\left(\mu_{i} I-K_{i}\right)^{\nu} q\left(K_{i}\right)$ where $q\left(K_{i}\right)$ is invertible. Hence $\left(\mu_{i} I-K_{i}\right)^{\nu}=$ 0 , so the operators $N_{i}:=\mu_{i} I-K_{i}$ are nilpotent for all $i=1,2, \ldots, n$. Note that

$$
T_{i}+K_{i}=\left(\mu_{i} I+T_{i}\right)+\left(K_{i}-\mu_{i} I\right)=\mu_{i} I+T_{i}-N_{i} .
$$

Since every $T_{i}$ is paranormal and hence algebraically paranormal, then $\mu_{i} I+T_{i}$ is algebraically paranormal, and hence by Theorem 2.4. $\mu_{i} I+T_{i} \in \mathcal{P}_{0}\left(Y_{i}\right)$. By Lemma 2.7 it then follows that $T_{i}+K_{i}=\left(\mu_{i} I+T_{i}\right)-N_{i} \in P_{0}\left(Y_{i}\right)$.

We show now that $T+K \in \mathcal{P}_{0}(H)$. Assume that $\lambda_{0} \in \pi_{00}(T+K)$. Then $\lambda_{0} \in$ iso $\sigma(T+K)$ and $\alpha\left(\lambda_{0} I-(T+K)\right)<\infty$. From equality (10) we have

$$
\left(\lambda_{0}-\mu_{i}\right) I-\left(T_{i}-N_{i}\right)=\lambda_{0} I-\left(T_{i}+K_{i}\right) .
$$

Fix $i \in \mathbb{N}$ such that $1 \leq i \leq n$. We distinguish two cases:

(I) $\left(\lambda_{0}-\mu_{i}\right) I-T_{i}$ is invertible.

(II) $\left(\lambda_{0}-\mu_{i}\right) I-T_{i}$ is not invertible.

In the first case, $N_{i}=\mu_{i} I-K_{i}$ being quasi-nilpotent and commuting with $\left(\lambda_{0}-\mu_{i}\right) I-T_{i}$, from the equivalence (1) we deduce that $\left(\lambda_{0}-\mu_{i}\right) I-T_{i}+N_{i}=$ $\lambda_{0} I-\left(T_{i}+K_{i}\right)$ is invertible and hence

$$
H_{0}\left(\lambda_{0} I-\left(T_{i}+K_{i}\right)\right)=\operatorname{ker}\left(\lambda_{0}-\left(T_{i}+K_{i}\right)\right)=\{0\} .
$$

In the second case we have $\lambda_{0}-\mu_{i} \in \sigma\left(T_{i}\right)$. We claim that $\lambda_{0} \in \pi_{00}\left(T_{i}+K_{i}\right)$. From (9), taking into account equality (10), we see that

$$
\lambda_{0} \in \text { iso } \sigma\left(T_{i}+K_{i}\right)=\text { iso } \sigma\left(\mu_{i} I+\left(T_{i}-N_{i}\right)\right),
$$

from which we obtain $\lambda_{0}-\mu_{i} \in$ iso $\sigma\left(T_{i}-N_{i}\right)$. Moreover, since $\alpha\left(\lambda_{0} I-(T+K)\right)<$ $\infty$, the inclusion

$$
\operatorname{ker}\left(\lambda_{0} I-\left(T_{i}+K_{i}\right)\right) \subseteq \operatorname{ker}\left(\lambda_{0} I-(T+K)\right)
$$

implies that $\alpha\left(\lambda_{0} I-\left(T_{i}+K_{i}\right)\right)=\alpha\left(\left(\lambda_{0}-\mu_{i}\right) I-\left(T_{i}-N_{i}\right)\right)<\infty$. Therefore $\lambda_{0}-\mu_{i} \in \pi_{0 \mathrm{f}}\left(T_{i}-N_{i}\right) \cap \sigma\left(T_{i}\right)$. Since $T_{i}$ is paranormal and $T_{i}$ satisfies Weyl's theorem, by Lemma 2.8 and Theorem 2.1 we then obtain that

$$
\lambda_{0}-\mu_{i} \in p_{00}\left(T_{i}\right)=\pi_{00}\left(T_{i}\right)=\sigma\left(T_{i}\right) \backslash \sigma_{\mathrm{w}}\left(T_{i}\right) .
$$

From equalities (11) we also have

$$
\sigma\left(T_{i}\right) \backslash \sigma_{\mathrm{w}}\left(T_{i}\right)=\sigma\left(T_{i}-N_{i}\right) \backslash \sigma_{\mathrm{w}}\left(T_{i}-N_{i}\right),
$$

so $\left(\lambda_{0}-\mu_{i}\right) I-\left(T_{i}-N_{i}\right)=\lambda_{0} I-\left(T_{i}+K_{i}\right)$ is Weyl. 
On the other hand, $\lambda_{0}-\mu_{i} \in \sigma\left(T_{i}\right)=\sigma\left(T_{i}-N_{i}\right)$; hence $\left(\lambda_{0}-\mu_{i}\right) I-\left(T_{i}-N_{i}\right)=$ $\lambda_{0} I-\left(T_{i}+K_{i}\right)$ is not invertible. This implies that $\alpha\left(\lambda_{0} I-\left(T_{i}+K_{i}\right)\right)>0$; otherwise, $\lambda_{0} I-\left(T_{i}+K_{i}\right)$ being Weyl we would have $\alpha\left(\lambda_{0} I-\left(T_{i}+K_{i}\right)\right)=\beta\left(\lambda_{0} I-\left(T_{i}+K_{i}\right)\right)=0$ and hence $\lambda_{0} \notin \sigma\left(T_{i}+K_{i}\right)$, contradicting (13).

Therefore, $\lambda_{0} \in \pi_{00}\left(T_{i}+K_{i}\right)$. Since $T_{i}+K_{i} \in \mathcal{P}_{0}\left(Y_{i}\right)$ we then conclude that there exists $\nu_{i} \in \mathbb{N}$ such that

$$
H_{0}\left(\lambda_{0} I-\left(T_{i}+K_{i}\right)\right)=\operatorname{ker}\left(\lambda_{0} I-\left(T_{i}+K_{i}\right)\right)^{\nu_{i}} .
$$

From the equality

$$
H_{0}\left(\lambda_{0} I-(T+K)\right)=\bigoplus_{i=1}^{n} H_{0}\left(\lambda_{0} I-\left(T_{i}+K_{i}\right)\right)
$$

and taking into account equalities (12) and (14), we then obtain

$$
\begin{aligned}
H_{0}\left(\lambda_{0} I-(T+K)\right) & =\bigoplus_{i=1}^{n} \operatorname{ker}\left(\lambda_{0} I-\left(T_{i}+K_{i}\right)\right)^{\nu_{i}} \\
& =\operatorname{ker}\left(\lambda_{0} I-(T+K)\right)^{\nu},
\end{aligned}
$$

where $\nu:=\max \left\{\nu_{1}, \ldots, \nu_{n}\right\}$. The last equalities hold for every $\lambda_{0} \in \pi_{00}(T+K)$, so $T+K \in \mathcal{P}_{0}(H)$.

We show now that $T+K$ has SVEP. Clearly, every $T_{i}$ has SVEP, since $T_{i}$ is paranormal. By Theorem 1.2 it then follows that all $T_{i}-N_{i}$ have SVEP, and hence every $T_{i}+K_{i}=\mu_{i} I+\left(T_{i}-N_{i}\right)$ has SVEP. From Theorem 2.9 of [1] we obtain that

$$
T+K=\bigoplus_{i=1}^{n}\left(T_{i}+K_{i}\right)
$$

has SVEP. By Theorem 2.2 we then conclude that $T+K$ satisfies Weyl's theorem, as desired.

It is well known that if for an operator $F \in L(X)$ there exists a positive integer $n$ for which $F^{n}$ is finite-dimensional, then $F$ is algebraic.

Corollary 2.9. If $T \in L(H)$ is paranormal and $F$ is an operator that commutes with $T$ and such that $F^{n}$ is a finite-dimensional operator for some positive integer $n$, then $T+F$ satisfies Weyl's theorem.

\section{ACKNOWLEDGMENTS}

The authors thank the referee for his several suggestions which have greatly contributed to improve the final form of this article.

\section{REFERENCES}

[1] P. Aiena Fredholm and local spectral theory, with application to multipliers. Kluwer Acad. Publishers (2004). MR2070395 (2005e:47001)

[2] P. Aiena Classes of Operators Satisfying a-Weyl's theorem, Studia Math.169 (2005), 105122. MR2140450(2006a:47005)

[3] P. Aiena, M. L. Colasante, M. Gonzalez Operators which have a closed quasi-nilpotent part, Proc. Amer. Math. Soc. 130, (9) (2002), 2701-2710. MR.1900878(2003g:47008)

[4] T. Ando Operators with a norm condition, Acta Sci. Math. (Szeged) 33 (1972), 169-178. MR 0320800 (47:9334)

[5] S. L. Campbell, B. C. Gupta On k-quasihyponormal operators, Math. Japon. 23, (1978-79), 185-189. MR0517798 (80b:47030) 
[6] N. N. Chourasia, P. B. Ramanujan Paranormal operators on Banach spaces, Bull. Austral. Math. Soc. 21 (1980), 161-168. MR0574835 (83a:47028)

[7] L. A. Coburn Weyl's theorem for nonnormal operators, Michigan Math. J. 20 (1970), 529544. MR0201969 (34:1846)

[8] R. E. Curto, Y. M. Han Weyl's theorem for algebraically paranormal operators, Int. Eq. Oper. Theory 47 (2003), 307-314. MR2012841(2004i:47041)

[9] B. P. Duggal Roots of contractions with Hilbert-Schmidt defect operators and $C_{0}$ completely non-unitary part, Comm. Math. Ann. Soc. Polonae 36 (1996), 85-106. MR1427824 (98a:47010)

[10] N. Dunford Spectral theory II. Resolution of the identity, Pacific J. Math. 2 (1952), 559-614. MR.0051435 (14:479a)

[11] N. Dunford Spectral operators, Pacific J. Math. 4 (1954), 321-354. MR0063563 (16:142d)

[12] J. K. Finch The single valued extension property on a Banach space, Pacific J. Math. 58 (1975), 61-69. MR0374985(51:11181)

[13] T. Furuta, M. Ito, T. Yamazaki A subclass of paranormal operators including class of log-hyponormal and several related classes, Scientiae Mathematicae 1 (1998), 389-403. MR 1688255 (2000d:47041)

[14] H. Heuser Functional Analysis. (1982), Wiley, New York. MR0640429 (83m:46001)

[15] K. B. Laursen, M. M. Neumann Introduction to local spectral theory, Clarendon Press, Oxford 2000. MR 1747914 (2001k:47002)

[16] K. K. Oberai On the Weyl spectrum II. Illinois J. Math. 21, 1, (1977), 84-90. MR0428073 $(55: 1102)$

[17] K. K. Oberai Spectral mapping theorem for essential spectra, Rev. Roum. Math. Pures et Appl. 25, 3, (1980), 365-373. MR0577924 (81j:47007)

[18] M. Oudghiri Weyl's and Browder's theorem for operators satysfying the SVEP, Studia Math. 163, 1, (2004), 85-101. MR2047466 (2005b:47006)

[19] M. Oudghiri Weyl's theorem and perturbations, Integral Equations Operator Theory 53 (2005), 535-545. MR2187437

Dipartimento di Matematica ed Applicazioni, Facoltà di Ingegneria, Università di Palermo, Viale delle Scienze, I-90128 Palermo, Italy

E-mail address: paiena@unipa.it

Departamento de Matemáticas, Facultad de Ciencias, Universidad UCla, Merida, VENEZUELA

E-mail address: rguillen@ula.ve 